\title{
Prediction of rebleeding from peptic ulcer experience with an endoscopic Doppler device
}

\author{
D E BECKLY AND M P CASEBOW \\ From the Departments of Radiology and Medical Physics, Plymouth General Hospital, Plymouth, Devon
}

SUMmaRY An endoscopic Doppler device has been used to predict which patients with bleeding peptic ulcers will require surgery to stop their bleeding. The results show that if a Doppler signal can be elicited from an ulcer then the likelihood of rebleeding sufficient to require surgery or lead to death is increased very significantly.

The widespread use of fibreoptic endoscopy in the diagnosis of upper gastrointestinal bleeding has failed to bring with it any great improvement in survival rates. ${ }^{1-3}$ This is not surprising as most mortality is caused either by rebleeding or by the sequelae of the surgery carried out to stop it. ${ }^{45}$ If diagnostic endoscopy is to affect mortality it must therefore either influence the incidence of rebleeding itself or contribute constructively to the criteria for surgery. Leaving aside the promising developments in therapeutic endoscopy such as lasers and heater probes, simple diagnostic endoscopy does not influence the incidence of rebleeding. There is evidence that careful attention to the endoscopic features of a peptic ulcer will allow some prediction of its likelihood of rebleeding. ${ }^{6-8}$ A prediction based on these criteria alone, however, may not be sufficiently accurate to influence the decision about surgery usefully. ${ }^{9}$ In an attempt to improve predictive accuracy we have developed a Doppler device which can be passed down the biopsy channel of the endoscope and will detect a patent artery in the base of an ulcer. The equipment has been described elsewhere $\mathrm{e}^{12}$ and similar equipment is now available commercially. The outside diameter of the transducer is $2.25 \mathrm{~mm}$ and it can be used with any forward or forward/oblique viewing endoscope having a biopsy channel size of $2.8 \mathrm{~mm}$ or greater. A bridge mechanism as on the Olympus GIFK2 is an advantage under some circumstances as it allows greater precision in the placement of the transducer. Side viewing endoscopes such as the JF1T or JF3.7 are also suitable.

Address for correspondence: Dr D E Beckly, Plymouth General Hospital, Radiodiagnostic Department, Freedom Fields, Plymouth PL4 $7 \mathrm{JJ}$.

Received for publication 19 April 1985

\section{Methods}

PATIENTS

During the period from October 1980 to December 1984 a total of 569 hospital inpatients were endoscoped in the Freedom Fields Hospital Endoscopy Unit because of haematemesis and/or melaena. Four hundred and twelve of these patients had peptic ulcers showing no stigmata of recent bleeding or were found to be bleeding from a lesion other than peptic ulcer. These were excluded from further study as our own experience and that of others ${ }^{6} 810$ shows that in the absence of stigmata the incidence of serious rebleeding is negligible. One hundred and sixty ulcers were found in the remaining 157 patients. There were five oesophageal ulcers, 80 gastric ulcers, three stomal ulcers, six pyloric canal ulcers and 66 duodenal ulcers. All these ulcers showed stigmata of recent haemorrhage. One hundred and eleven ulcers in 109 patients were excluded for the reasons shown in Table 1 . This left a total of

\section{Table 1 Exclusions}

\begin{tabular}{lc}
\hline & Cases (no) \\
\hline Ulcer laser coagulated as part of another trial & 43 \\
Endoscopy carried out by endoscopist not taking & 15 \\
$\quad$ part in trial & 13 \\
Paediatric endoscope used & 6 \\
Inaccessible site or whole ulcer not seen & 5 \\
Oesophageal ulcer (transmitted cardiac movements & \\
prevent use of Doppler) & 5 \\
Active bleeding obscures lesion & 22 \\
Miscellaneous (scope failure, Doppler unavailable, & \\
patient collapse etc) & 109 \\
\end{tabular}


49 ulcers in 48 patients and these were studied further. Age, sex, ulcer sites, and types of stigmata for the excluded cases and the study cases are in all respects similar and are shown in Tables 2 and 3.

Diagnostic endoscopy was carried out on the next routine list after admission unless the patient's condition caused concern when endoscopy was done as an emergency. Most patients were endoscoped within 24 hours of their admission. Endoscopy was carried out with an Olympus GIFK2 and if a peptic ulcer was found the ulcer base was washed with water through a washing catheter before assessment of visual stigmata was made. Each ulcer was assigned to one of the following groups: (1) Dark stains, (defined as dark brown or black stains not raised aboye the level of the slough). (2) Adherent clot, (defined as 'organised' clot that will not wash off the ulcer base). (3) 'Visible vessel', (defined as a small dark spot definitely raised above the level of the slough).

If more than one of the above features were present the ulcer was graded as showing the stigma with the higher number. The Doppler transducer was then passed via the biopsy channel of the endoscope and worked systematically across the surface of the ulcer. Care was taken to cover as much of the ulcer base as possible but in some cases coverage was incomplete because of the position of the ulcer. A positive response was recorded if an unequivocal pulsatile sound could be heard in the headphones in time with the patient's pulse. In order to distinguish between a signal coming from a deep structure such as the aorta and an artery actually in the ulcer base a positive response was only recorded if the signal was localised to a small area and could be made to disappear with slight movements of the transducer (Fig. 1). Details of the endoscopy findings including the type of visual stigmata present were made available to the referring clinical team but the Doppler findings were withheld. The patient was then managed according to the policy of the clinical team concerned. After the patient's discharge from hospital or death the notes were reviewed. If the patient had undergone

Table 2 Excluded $v$ study cases (site)

\begin{tabular}{lrrrr}
\hline Site & \multicolumn{2}{c}{ Excluded cases (\%) } & \multicolumn{2}{c}{ Study cases (\%) } \\
\hline Oesophageal & 5 & $(5)$ & - & - \\
Gastric & 54 & $(49)$ & 26 & $(53)$ \\
Stomal & 2 & $(2)$ & 1 & $(2)$ \\
Pyloric canal & 4 & $(4)$ & 2 & $(4)$ \\
Duodenal & 46 & $(40)$ & 20 & $(41)$ \\
Total & 111 & 71 & 49 & 68 \\
Mean age (yr) & \multicolumn{3}{c}{1.24} & \multicolumn{3}{c}{1.52} \\
Male/female ratio & \multicolumn{2}{c}{1.52} \\
\hline
\end{tabular}

Table 3 Excluded $v$ study cases (stigmata)

\begin{tabular}{lcccc}
\hline Stigmata & \multicolumn{3}{c}{ Excluded cases (\%) } & Study cases (\%) \\
\hline Dark stains & 35 & $(31)$ & 20 & $(41)$ \\
Fresh clot & 44 & $(40)$ & 20 & $(41)$ \\
Visible vessel & 18 & $(16)$ & 9 & $(18)$ \\
Active bleed & 14 & $(13)$ & - & - \\
\hline
\end{tabular}

surgery it was determined whether this was undertaken solely to stop haemorrhage or whether there had been any other indication. If the patient had died it was determined whether death was directly related to gastrointestinal bleeding. Only those cases in whom surgery was carried out solely to stop haemorrhage or who bled to death were classified as 'rebleeds'.

\section{Results}

Nine patients were operated on for recurrent bleeding. In five cases (two gastric ulcers, three duodenal ulcers) recurrent bleeding was confirmed at the site of a Doppler positive ulcer. In the remaining four operated cases the Doppler examination was negative. Two cases were found to be bleeding from a Doppler negative ulcer and represent false negative examinations. In one case bleeding was found to be coming from oesophageal varices which coexisted with a Doppler negative duodenal ulcer. In another case although operation was carried out on account of supposed rebleeding no evidence of recent bleeding was found. Both these latter cases were classified as not rebleeding. Three patients died of rebleeding. In all three cases this was confirmed at necropsy as coming from Doppler positive duodenal ulcers. One patient had refused surgery, one had been judged unfit for

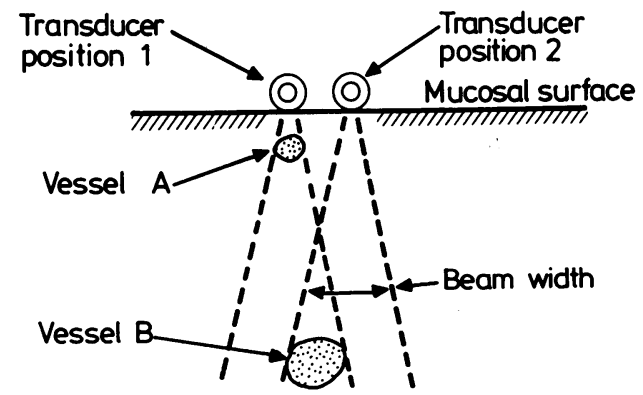

Fig. 1 When the cylindrical transducer, seen end on, is moved from position 1 to position 2 the signal from a superficially placed vessel $A$ is lost but that from a large deeper vessel $B$ is still present. 
surgery and the third died before operation could be performed. Thus a total of 10 cases rebled from a confirmed site and in eight of these the ulcer was Doppler positive and in two, Doppler negative. Four other patients underwent surgery. In two cases this was done for obstruction, in one case for perforation, and in one semi-elective surgery was carried out for repeated episodes of pain and melaena. There were five deaths in all; four were from bleeding, one of whom had been operated on. One death was from an unrelated cause. Thirty nine ulcers in 38 patients did not rebleed and all but three of these ulcers were Doppler negative. The fraction rebleeding in each stigma group is shown in Table 4. No case with simple dark stains rebled and none were Doppler positive. In the fresh clot group and in the visible vessel group the presence of a positive Doppler response enhances the likelihood of rebleeding to a statistically significant level using the Fisher exact probability test. Thus in general an ulcer with adherent fresh clot had a $30 \%$ chance of rebleeding. If this ulcer was Doppler positive its chances of rebleeding rose to $67 \%$. Whereas if it was Doppler negative the chances fell to $14 \%$. In the case of an ulcer with a visible vessel the overall risk was $44 \%$. If the ulcer was Doppler positive this rose to $80 \%$. None of the four Doppler negative cases with visible vessels rebled. When these groups are combined the results are highly significant $\mathrm{p}<2 \times 10^{-5}$. The presence or absence of a Doppler signal correctly predicts the final outcome in 44 of the 49 patients $(90 \%)$. The sensitivity of the test given by the proportion of true positives is eight of $11(73 \%)$ and the specificity, the proportion of true negatives is 36 of 38 (95\%) (Fig. 2).

\section{Discussion}

It is of interest that the majority of ulcers are Doppler negative in spite of the fact that they are assumed to have been the site of a recent bleed. This is probably because the initial event of erosion into the artery resulted in first bleeding and then spontaneous thrombosis back to the next branch. This would accord well with the findings that Doppler negative ulcers have a low risk of re-

Table 4 Fraction rebleeding

\begin{tabular}{lllll}
\hline Stigma & $\begin{array}{l}\text { Doppler } \\
+v e\end{array}$ & $\begin{array}{l}\text { Doppler } \\
-v e\end{array}$ & Total & $P$ \\
\hline Dark stain & - & $0 / 20$ & $0 / 20$ & - \\
Fresh clot & $4 / 6(67 \%)$ & $2 / 14(14 \%)$ & $6 / 20(30 \%)$ & $0 \cdot 037$ \\
Visible vessel & $4 / 5(80 \%)$ & $0 / 4(00 \%)$ & $4 / 9(44 \%)$ & $0 \cdot 040$ \\
Total & $8 / 11(73 \%)$ & $2 / 38(5 \%)$ & $10 / 49(20 \%)$ & $2 \times 10^{-5}$ \\
\hline
\end{tabular}

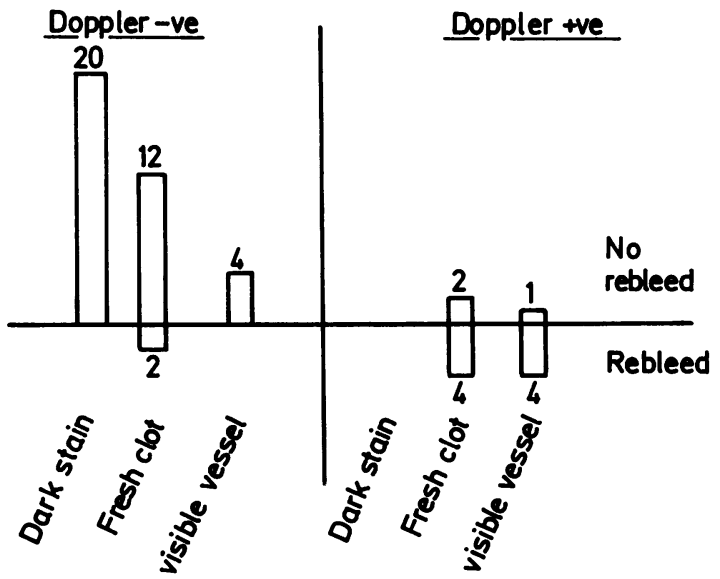

Fig. 2 The Doppler status of each case was determined by the presence or absence of a signal localised to the ulcer base and in time with the patient's pulse. Rebleeding was defined as recurrence of bleeding sufficient to require surgery or result in the patient's death.

bleeding.

A Doppler signal may occasionally be found in the absence of visual stigmata and is then assumed to represent continuing flow in a parent vessel from which a branch has bled and thrombosed. The false negative results in two cases are probably because of technical failure to elicit a Doppler response due to poor transducer contact with the ulcer base. Our results suggest that the routine use of an endoscopic Doppler probe in cases of bleeding peptic ulcer is helpful in predicting which cases will rebleed sufficiently to require surgery. If the results are confirmed with greater numbers of patients then a useful advance will have been made. Both the selection for, and timing of, surgery will be potentially improved. This in turn might be expected to result in improved mortality and morbidity. The Doppler device is also thought to have a useful role when used in conjunction with a laser or other endoscopic method of haemostasis as it allows identification of the exact site of the underlying artery and can be used to determine if and when flow ceases. A possible development in this context would be the use of pulsed Doppler which although considerably more expensive allows range gating. This could be used to measure the depths at which the signal appears and disappears and thus give a measure of the vessel size provided of course that the beam is normal, or nearly normal, to the long axis of the vessel.

Finally the endoscopic Doppler has been used by us to determine whether an artery lies in the line of intended cut at endoscopic sphincterotomy. This 
may be particularly useful when the question of extending an existing sphincterotomy arises. In these circumstances the transducer can be easily placed within the bile duct as well as against the duodenal mucosa.

\section{References}

1 Winans CS. Emergency upper gastrointestinal endoscopy: does haste make waste? Am J Dig Dis 1977; 22: 536-40.

2 Eastwood GL. Does early endoscopy benefit the patient with upper gastrointestinal bleeding? Gastroenterology 1977; 72: 737-9.

3 Editorial. Bleeding ulcers: scope for improvement? Lancet 1984; 1: 715-6.

4 Logan RFA, Finlayson NDC. Death in acute upper gastrointestinal bleeding. Can endoscopy reduce mortality? Lancet 1976; 1: 1173-5.

5 Allan R, Dykes P. A study of the factors influencing mortality rates from gastrointestinal haemorrhage. $Q J$ Med 1976; 180: 533-50.

6 Foster DN, Miloszewski KJA, Losowsky MS. Stigmata of recent haemorrhage in diagnosis and prognosis of upper gastrointestinal bleeding. Br Med J 1978; 1: 1173-7.

7 Griffiths WJ, Neumann DA, Welsh JD. The visible vessel as an indicator or uncontrolled or recurrent gastrointestinal haemorrhage. N Engl J Med 1979; 300: 1411-3.

8 Storey DW, Bown SG, Swain CP, Salmon PR, Kirkham JS, Northfield TC. Endoscopic prediction of recurrent bleeding in peptic ulcers. $N$ Engl J Med 1981; 305: $915-6$.

9 MacLeod IA, Mills PR, MacKenzie JF, Joffe SN, Russell RI, Carter DC. Neodymium yttrium aluminium garnet laser photocoagulation for major haemorrhage from peptic ulcers and single vessels: a single blind controlled study. Br Med J 1983; 286: 345-8.

10 Swain CP, Bown SG, Storey DW, Kirkham JS, Northfield TC, Salmon PR. Controlled trial of Argon laser photocoagulation in bleeding peptic ulcers. Lancet 1981; 2: 1313-6.

11 Vantrappen G, Rutgeerts P, Broeckaert L, Janssens J, Coremans G. Controlled trial of Nd YAG laser treatment for upper digestive haemorrhage. [Abstract] Gastroenterology 1981; 80: A-57.

12 Beckly DE, Casebow MP, Pettengell KE. The use of a Doppler Ultrasound probe for localising arterial blood flow during upper gastrointestinal endoscopy. Endoscopy 1982; 14: 146-6. 